\title{
Petri Net Based Modeling and Correctness Verification of Collaborative Emergency Response Processes
}

\author{
Cong Liu ${ }^{1,2}$, Feng Zhang ${ }^{1}$ \\ ${ }^{1}$ Shandong University of Science and Technology, Qingdao, 266590 China \\ ${ }^{2}$ Eindhoven University of Technology, 5600MB Netherland \\ E-mails: liucongchina@163.com_zhangfengsdkd@163.com
}

\begin{abstract}
Petri net extended with resource and message factors, RMWF-Net for short, is proposed to model collaborative emergency response processes with various collaboration patterns. Correctness of a RMWF-Net is formally defined and verified using reachability analysis. A running case of collaborative fire emergency response processes is given to validate the approach.
\end{abstract}

Keywords: Collaborative emergency management, emergency process modeling, collaboration patterns, correctness verification, Petri net.

\section{Introduction}

Major industrial emergencies and accidents, such as fire, explosion or personnel poisoning accidents in smelting industry, may cause immediate risks and losses to human health, life and property, which require urgent interventions to prevent its degradation. These interventions are organized as a process that is usually described in a pre-established emergency plan [12, 23]. It arranges the structure of involved emergency organization, personals, technology and equipment, material resources, commands and inter-organization collaborations beforehand. Unfortunately, it is difficult to judge if these emergency plans are reasonable or well-coordinated before implemented. Therefore, evaluation and verification of emergency plans (or emergency exercise) is becoming more and more important to emergency mission success $[6,17,22]$.

The evaluation and verification of emergency response plan has been researched in many industries and fields, such as the chemical industry [19, 21, 23], the nuclear industry [5,9], the water pollution emergency [3, 7], the earthquake emergency [1, 4], and so on [2, 8, 10, 12-14, 17]. However, most of these focus on emergency plan involving only single emergency organization, and no crossorganization collaborations are investigated. Actually, to effectively cope with an emergency several emergency response organizations need to collaborate with each other to achieve the whole mission in a rapid and efficient manner. In this case, 
designing and verifying such a cross-organizational collaborative emergency response processes are really complicated and time-consuming. As tools of modeling and analyzing physical systems, Petri nets [11, 15-16, 18, 25-29] have shown great power in dealing with concurrencies and conflicts. Therefore, it has been widely used to model and analyze emergency response processes $[9,11,21$ 23]. Towards the collaborative emergency management, Guo and $\mathrm{Kapucu}$ [6] examined coordination in disaster response by simulation using Petri net focusing on inter-organizational process, and find some useful insights on the performance assessments. However, this work does not mention how to model a collaborative emergency response process. Meng et al. [14] used Petri net to model Urban Emergency Response Systems by defining four kinds of cross-organization task coordination patterns. However, no further formal verification analysis is defined for a collaborative emergency response process model. To move a step further, we mainly discuss a systematic modeling and verification of collaborative emergency response processes using Petri net formulism in this paper. To explicitly characterize the collaboration features among different emergency organizations, a series of collaboration patterns, including message exchange pattern, resource sharing pattern, and task synchronization pattern, are formally defined on organization level. Based on these collaboration patterns an integrated collaborative emergency response process can be obtained. In addition, correctness verification criteria are also investigated to check the correctness of a collaborative emergency response process model.

The remainder of this paper is organized as follows. Section 2 introduces a collaborative fire emergency response processes case study. In Section 3, RMWFnet based modeling approaches of emergency response process for single emergency organization is discussed. Section 4 mainly presents a series of collaboration patterns, based on which RMWF-net model of collaborative emergency response processes is obtained. In Section 5, we formally define the correctness criteria and its corresponding verification method for the collaborative emergency response processes using RMWF-net. Finally, Section 6 draws concluding remarks.

\section{A collaborative fire emergency response processes case study}

This section presents a simple collaborative emergency response process to cope with a fire accident, based on which our modeling and verification approach is demonstrated in detail. Some of the critical missions are (1) rescue of victims; and (2) disposal of the fire. This emergency response scenario involves three organizations: Emergency Command Center (ECC), fire brigade and hospital.

Its detailed emergency planning process includes: (1) after receiving the fire emergency information, the ECC first informs the hospital to perform medical rescue, and the fire brigade to conduct fire disposal; (2) the fire brigade rushes to the site once the fire rescue instruction from the ECC has been received, and conducts its specific disposal activities, and finally reports the fire disposal results; (3) the hospital personnel rushes to the site once the medical rescue instruction from 
the ECC has been received, and conducts its specific disposal activities, and finally reports the medical rescue results; (4) after receiving both feedback information from hospital and fire brigade, the ECC makes emergency summary and evaluation, and finally do the file archive; and (5) finally, the ECC, hospital, and the fire brigade do the media coverage together.

According to the above-mentioned descriptions, activity information of the ECC, fire brigade, and hospital are shown in Tables 1-3. And Table 4 describes the meaning of the involved message and resource symbols.

Table 1. Tasks and causalities information of the Emergency Command Center

\begin{tabular}{|c|c|c|c|c|c|}
\hline Task ID & Task name & $\begin{array}{c}\text { Required } \\
\text { messages }\end{array}$ & $\begin{array}{c}\text { Sent } \\
\text { messages }\end{array}$ & $\begin{array}{c}\text { Required } \\
\text { resource }\end{array}$ & Pre-tasks \\
\hline$t_{1}$ & $\begin{array}{c}\text { "Receive the emergency } \\
\text { information" }\end{array}$ & $\varnothing$ & $\varnothing$ & $\varnothing$ & $\{\varnothing\}$ \\
\hline$t_{2}$ & $\begin{array}{c}\text { "inform the hospital } \\
\text { and fire brigade" }\end{array}$ & $\varnothing$ & $\left\{p_{m 3}, p_{m 4}\right\}$ & $\varnothing$ & $\left\{t_{1}\right\}$ \\
\hline$t_{3}$ & $\begin{array}{c}\text { "Emergency summary } \\
\text { and evaluation" }\end{array}$ & $\left\{p_{m 6}, p_{m 7}\right\}$ & $\varnothing$ & $\varnothing$ & $\left\{t_{2}\right\}$ \\
\hline$t_{4}$ & "File archive" & $\varnothing$ & $\varnothing$ & $\varnothing$ & $\left\{t_{3}\right\}$ \\
\hline$t_{5}$ & "Do media coverage" & $\varnothing$ & $\varnothing$ & $\varnothing$ & $\left\{t_{4}\right\}$ \\
\hline
\end{tabular}

Table 2. Tasks and causalities information of the Fire Brigade

\begin{tabular}{|c|c|c|c|c|c|}
\hline \multirow{2}{*}{ Task ID } & Task name & $\begin{array}{c}\text { Required } \\
\text { messages }\end{array}$ & $\begin{array}{c}\text { Sent } \\
\text { messages }\end{array}$ & $\begin{array}{c}\text { Required } \\
\text { resource }\end{array}$ & Pre-tasks \\
\hline$t_{6}$ & "Rush to the site" & $\left\{p_{m 1}\right\}$ & $\varnothing$ & $\left\{p_{r}\right\}$ & $\{\varnothing\}$ \\
\hline$t_{7}$ & "Fight the fire" & $\varnothing$ & $\varnothing$ & $\varnothing$ & $\left\{t_{6}\right\}$ \\
\hline$t_{8}$ & "Recovery the site" & $\varnothing$ & $\varnothing$ & $\varnothing$ & $\left\{t_{7}\right\}$ \\
\hline$t_{9}$ & "Conduct mitigation operations" & $\varnothing$ & $\left\{p_{m 3}\right\}$ & $\varnothing$ & $\left\{t_{7}\right\}$ \\
\hline$t_{10}$ & "Report the fire rescue" & $\varnothing$ & $\varnothing$ & $\varnothing$ & $\left\{t_{8}, t_{9}\right\}$ \\
\hline$t_{5}$ & "Do media coverage" & $\varnothing$ & $\varnothing$ & $\varnothing$ & $\left\{t_{10}\right\}$ \\
\hline
\end{tabular}

Table 3. Tasks and causalities information of the Hospital

\begin{tabular}{|c|c|c|c|c|c|}
\hline Task ID & Task name & $\begin{array}{c}\text { Required } \\
\text { messages }\end{array}$ & $\begin{array}{c}\text { Sent } \\
\text { messages }\end{array}$ & $\begin{array}{c}\text { Required } \\
\text { resource }\end{array}$ & Pre-task \\
\hline$t_{11}$ & "Rush to the site" & $\left\{p_{m 1}\right\}$ & $\varnothing$ & $\left\{p_{r}\right\}$ & $\{\varnothing\}$ \\
\hline$t_{12}$ & "Shunt the wounded" & $\varnothing$ & $\varnothing$ & $\varnothing$ & $\left\{t_{11}\right\}$ \\
\hline$t_{13}$ & "Treat the severely injured people" & $\varnothing$ & $\varnothing$ & $\varnothing$ & $\left\{t_{12}\right\}$ \\
\hline$t_{14}$ & "Treat the slightly injured people" & $\varnothing$ & $\varnothing$ & $\varnothing$ & $\left\{t_{12}\right\}$ \\
\hline$t_{15}$ & "Environmental quality detection" & $\varnothing$ & $\varnothing$ & $\varnothing$ & $\left\{t_{13}, t_{14}\right\}$ \\
\hline$t_{16}$ & "Report the medical rescue" & $\varnothing$ & $\left\{p_{m 4}\right\}$ & $\varnothing$ & $\left\{t_{15}\right\}$ \\
\hline$t_{5}$ & "Do media coverage" & $\varnothing$ & $\varnothing$ & $\varnothing$ & $\left\{t_{16}\right\}$ \\
\hline
\end{tabular}

Table 4. Message and resource information

\begin{tabular}{|c|c|}
\hline Symbol & Meaning \\
\hline$p_{m 1}$ & fire rescue instruction \\
\hline$p_{m 2}$ & medical rescue instruction \\
\hline$p_{m 3}$ & fire rescue results \\
\hline$p_{m 4}$ & medical rescue results \\
\hline$p_{r}$ & public transportation vehicle \\
\hline
\end{tabular}


Based on the preceding running case, we have the following explanations for Tables 1-3.

(1) This fire emergency response process involved three emergency organizations: the ECC, fire brigade and hospital. Each organization has its private response process and task set. For example, the business of the ECC is composed of five sequence activities which are "receive the emergency information", "inform the hospital and fire brigade", "emergency summary and evaluation", "file archive" and "do media coverage".

(2) Each emergency task contains several components. Generally speaking, an activity contains activity name, required messages, sent messages, pre-activities, and resource requirements. For example, to perform the activity "Rush to the site" in hospital, the message "fire rescue instruction" and resource "public transportation vehicle" are required.

(3) These emergency organizations need to collaborate with one another to accomplish the whole emergency mission. The collaboration includes the following three scenarios: (a) one organization requires the message information sent by the other organization to launch or initialize its own emergency process; (b) several organizations need to synchronize to complete certain emergency task; and (c) emergency resources may be shared among different organizations, i.e. emergency organizations should coordinated with each other to use them.

\section{Petri net based modeling of collaborative emergency response processes}

In this section, RMWF-net (Resource and Message Work Flow net), a special kind of Petri net extended with resource and message information, is proposed to model the collaborative emergency response processes.

\subsection{RMWF-net}

Tasks are key elements of emergency response processes. An emergency task is composed of task content, required message set, sent message set, and required resource set. In this way, formal specification of an emergency task is given in Definition 3.1.

Definition 3.1. An emergency task is a 5-tuple EmergencyTask=<TID, TName, MReq, MSent, RSet>, where

(1) TID is the unified identifier of an emergency task;

(2) TName represents the content an emergency task;

(3) MReq is the message set that is required when an emergency task starts;

(4) MSent is the message set that is sent when an emergency task ends; and

(5) RSet is the resource set that is required to execute an emergency task.

Considering for example, emergency task $t_{11}$ in can be formulated as: 〈" $t_{11}$ ", "Rush to the site", $\left.\left\{p_{m 2}\right\}, \varnothing,\left\{p_{r}\right\}\right\rangle$. During the execution of $t_{11}$, it requires a message $p_{m 2}$, and accesses a resource $p_{r}$. 
In this paper, our work is based on Petri nets, WF-nets (Work Flow nets) to be more accurate. We assume that readers are familiar with the basics of Petri nets. Some of the essential terminologies and notations of WF-net [20] and Petri nets [11, $15,16,18]$ are given for self-completeness.

Definition 3.2. A Petri net is a 4-tuple $\Sigma=\left(P, T ; \quad F, M_{0}\right)$, where (1) $P=\left\{p_{1}, p_{2}, \ldots, p_{m}\right\}$ is a finite set of places; (2) $T=\left\{t_{1}, t_{2}, \ldots, t_{n}\right\}$ is a finite set of transitions; (3) $F \subseteq(P \times T) \cup(T \times P)$ is a finite set of arcs (flow relation); (4) $M_{0}: P \rightarrow\{0,1,2,3, \ldots\}$ is the initial marking; (5) $P \cap T=\varnothing$ and $P \cup T \neq \varnothing$.

For all $x \in P \cup T$, the set $x=\{y \mid y \in P \cup T \wedge(y, x) \in F\}$ is the pre-set of $x$, and $x^{\bullet}=\{y \mid y \in P \cup T \wedge(x, y) \in F\}$ is the post-set of $x ; p$ is marked by $M$ iff $M(p)>0$. A transition $t \in T$ is enabled under $M$, if and only if $\forall p \in{ }^{\bullet} t: M(p)>0$, denoted as $M[t>$. If $M\left[t>\right.$ holds, $t$ may fire, resulting in a new marking $M^{\prime}$, denoted as $M\left[t>M^{\prime}\right.$, such that $M^{\prime}(p)=M(p)-1$ if $\forall p \in \in^{\bullet} \backslash t^{\bullet}, M^{\prime}(p)=M(p)+1$ if $\forall p \in t^{\bullet} \backslash{ }^{\bullet} t$, and otherwise $M^{\prime}(p)=M(p)$. An initial marking is denoted by $M_{0}$ and $R\left(M_{0}\right)$ is defined as the set of all reachable marking set of $\Sigma$ where $\forall M_{i} \in R\left(M_{0}\right)$ such that $M_{0}[\delta>M$.

A Petri net which models a workflow process is called the work flow net whose definition is briefly reviewed due to [20].

Definition 3.3. A Petri net $\Sigma=\left(P, T ; F, M_{0}\right)$ is a WF-net if: (1) there is one source place $p_{\mathrm{s}} \in P$ such that $p_{\mathrm{s}}=\varnothing$; (2) there is one sink place $p_{\mathrm{e}} \in P$ such that $p_{\mathrm{e}}{ }^{\bullet}=\varnothing$; (3) each node $x \in P \cup T$ is on a path from $p_{\mathrm{s}}$ to $p_{\mathrm{e}}$; and (4) $\forall p \in P, M_{0}(p)=1$ if $p=p_{\mathrm{s}}$, and otherwise $M_{0}(p)=0$.

In a WF-net, the transition set $T$ is used to represent the normal task in a workflow, the place set $P$ is used to represent logic connection relation of tasks, and source place and sink place specially represent the start and end of the process respectively. Based on the classical WF-net, we propose RMWF-net by extending it with Resource and Message information to model the collaborative emergency response processes.

Definition 3.4. Given a workflow net $\Sigma=\left(P, T ; F, M_{0}\right)$, it is a RMWF-net if:

(1) $P=P_{\mathrm{L}} \cup P_{\mathrm{R}} \cup P_{\mathrm{M}}, P_{\mathrm{L}} \cap P_{\mathrm{R}}=\varnothing, P_{\mathrm{R}} \cap P_{\mathrm{M}}=\varnothing, P_{\mathrm{L}} \cap P_{\mathrm{M}}=\varnothing$; $P_{\mathrm{L}}$ represents the logic place set, $P_{\mathrm{M}}$ represents the message exchange place set, and $P_{\mathrm{R}}$ represents resource sharing place set in $\Sigma_{\mathrm{TRM}}$;

(2) $F=F_{\mathrm{L}} \cup F_{\mathrm{R}} \cup F_{\mathrm{M}}$, where

(3.1) $F_{\mathrm{L}}=\left(P_{\mathrm{L}} \times T\right) \cup\left(T \times P_{\mathrm{L}}\right)$ represents the logical structure of the model;

(3.2) $F_{\mathrm{R}}=\left(P_{\mathrm{R}} \times T\right) \cup\left(T \times P_{\mathrm{R}}\right)$ represents the required resource relations; and

(3.3) $F_{\mathrm{M}}=\left(P_{\mathrm{M}} \times T\right) \cup\left(T \times P_{\mathrm{M}}\right)$ represents the required and sent message relations; and

(3) $\forall p \in P, M_{0}(p)=1$ if $p \in P_{\mathrm{R}}$ or ${ }^{\bullet} p=\varnothing$, and otherwise $M_{0}(p)=0$.

The firing rule of a RMWF-net is same as that of a traditional WF-net. Given a marking $M \forall t \in T, t$ is enabled under $M$ if $\forall p \in{ }^{\bullet} t, M(p) \geq 1$. Firing an enabled $t$ removes a token from each of places in ${ }^{\circ} t$ and deposits one to each place in $t^{\circ}$. All properties, such as reachability, boundedness, etc., can be defined similarly. The main difference between a RMWF-net and a WF-net is that the RMWF-net is a special kind of WF-net extended with resource place set $\left(P_{\mathrm{R}}\right)$ and message place set $\left(P_{\mathrm{M}}\right)$. 
3.2. RMWF-net based modeling of single organization emergency response process

Modeling a single organization emergency response process involves the following two steps: (1) modeling emergency tasks with RMWF-net; and (2) modeling control structure with RMWF-net.

In a RMWF-net, an emergency task is represented by a transition which has one input logic place and one output logic place representing the start and end state respectively. In addition, to represent the involved resources and messages, the corresponding places are added with flow relations. An example emergency task modeled with RMWF-net is illustrated in Fig. 1 , where $p_{\text {ready }}$ is the ready place, $p_{\text {end }}$ is the end place, $p_{\mathrm{r}}$ is its resource place, and $p_{\text {messageReq }}$ and $p_{\text {messagesent }}$ are corresponding message places. A logic place is drawn with a normal circle, a double circle with full line is used to represent a resource place, and a circle with dash line is used to represent a message place.

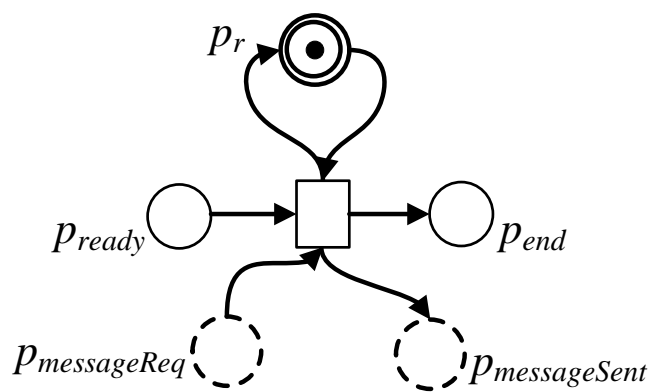

Fig. 1. An Example Emergency Task Model in RMWF-net

In this section, emergency task dependencies within one single emergency organization are first investigated to build the control-flow structure. It is worth noting that the control structure of an RMWF-net, denoted as $\left(P_{L}, T ; F_{L},\left.M_{0}\right|_{L}\right)$ where $\left.M_{0}\right|_{L}$ is the projection of $M_{0}$ on $P_{\mathrm{L}}$, is actually a standard WF-net. This way, its basic control structures, including sequence structure, concurrent structure, choice structure, and loop structure, can be modeled similarly as introduced in [25]. Based on the modeling approaches of basic control-flow routings, a WF-net model is constructed to express task dependencies. Then, we add resource places, message places and corresponding flow relations to its corresponding emergency tasks. In this way, the RMWF-net is obtained to model the emergency response process of a single organization. Considering for example the fire emergency scenario in Section 2, the RMWF-net models of the ECC, fire brigade, and hospital are obtained and shown in Table 5.

By detailed inspecting the RMWF-net model of each emergency organization, we argue that: (1) one organization requires the message sent by the other organization to launch or initialize its own emergency process; (2) some emergency tasks are synchronized among different emergency organizations, i.e., they work together to complete certain emergency task; and (3) emergency resources are shared among different organizations. In view of these features of a collaborative 
emergency response process, we need to investigate several collaboration patterns to present an accurate modeling approach.

Table 5. RMWF-net models of emergency organizations

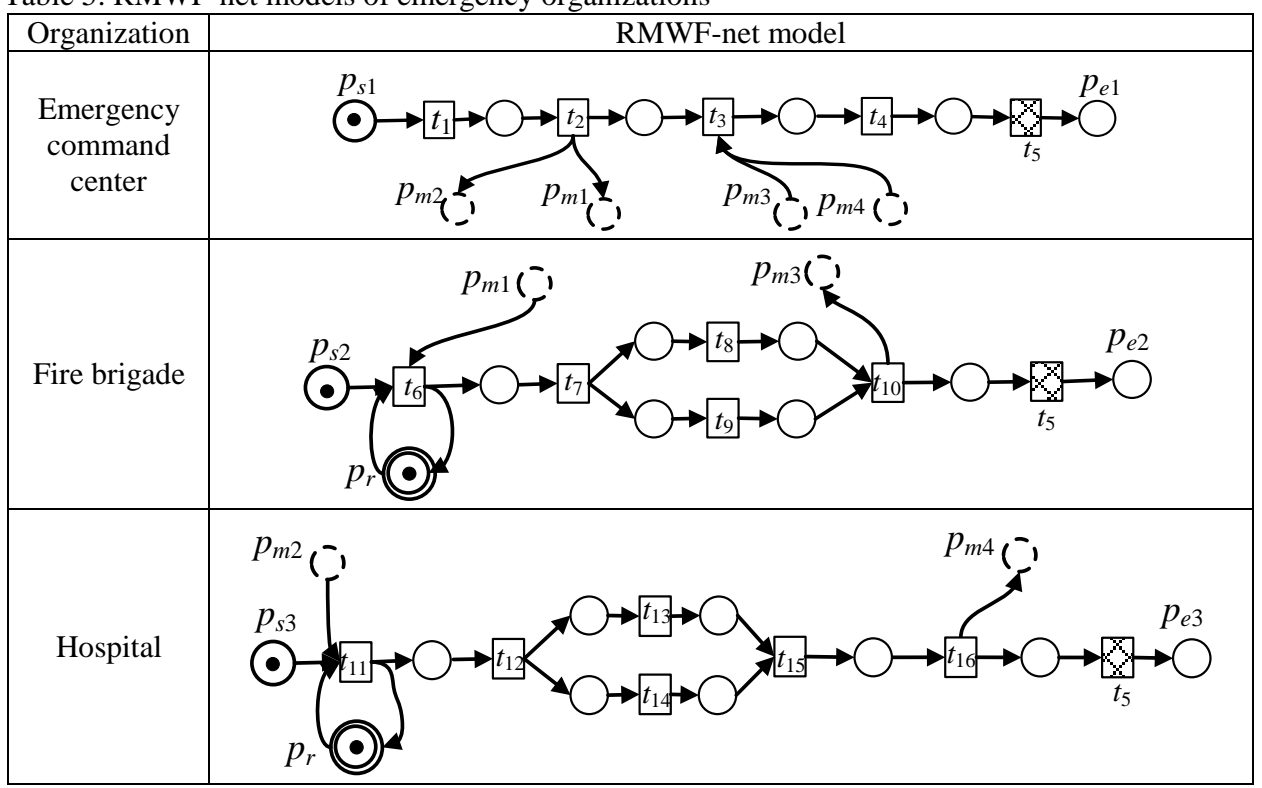

\section{Modeling approaches for collaborative emergency response processes}

In this section, we first address different kinds of collaboration patterns among emergency organizations. And then RMWF-net model of collaborative emergency response processes is obtained on the basis of these collaboration patterns.

\subsection{Taxonomy of collaboration patterns among different emergency organizations}

In this subsection, taxonomy of collaboration patterns [25], [26] among different emergency organizations is formally defined first, which includes message exchange pattern, resource sharing pattern and task synchronization pattern. Here we only define these collaboration patterns between two organizations, and it is easy to extend them to the case of an arbitrary number.

\subsubsection{Message exchange pattern}

If the received/sent message sets of two emergency organizations interact completely or partially, it is defined that a message exchange pattern exists among them. Its formal definition and RMWF-net based modeling methods are presented in the following definitions.

Definition 4.1. A message exchange pattern is a 5-tuple $\mathrm{MEP}=\langle\mathrm{PID}$, Orgs, Mele, STID, RTID $\rangle$, where

(1) PID is the unified identifier of a pattern; 
(2) Orgs represents the organizations involved in this pattern;

(3) Mele is the exchanged message element;

(4) STID is the emergency task ID that sends the message element; and

(5) RTID is the emergency task ID that receives the message element.

Definition 4.2. Let $\Sigma_{\mathrm{RM} 1}=\left(P_{1}, T_{1} ; F_{1}, M_{01}\right)$ and $\Sigma_{\mathrm{RM} 2}=\left(P_{2}, T_{2} ; F_{2}, M_{02}\right)$ be the RMWF-net of two emergency organizations, where $P_{1}=P_{\mathrm{L} 1} \cup P_{\mathrm{R} 1} \cup P_{\mathrm{M} 1}$, $P_{2}=P_{\mathrm{L} 2} \cup P_{\mathrm{R} 2} \cup P_{\mathrm{M} 2}$. If $P_{\mathrm{M} 1} \cap P_{\mathrm{M} 2} \neq \varnothing$, then a message exchange pattern exists between them.

Considering for example, a message exchange pattern which is formulated as: $\left\langle\right.$ " $P_{1}$ ", $\left.\left\{O_{1}, O_{2}\right\},\left\{p_{m 1}\right\},\left\{t_{2}\right\},\left\{t_{6}\right\}\right\rangle$. It involves two emergency organizations $O_{1}$ and $O_{2}$, and task $t_{2}$ sends message $p_{m 1}$ and task $t_{6}$ receives it. This message exchange pattern is illustrated in Fig. 2.

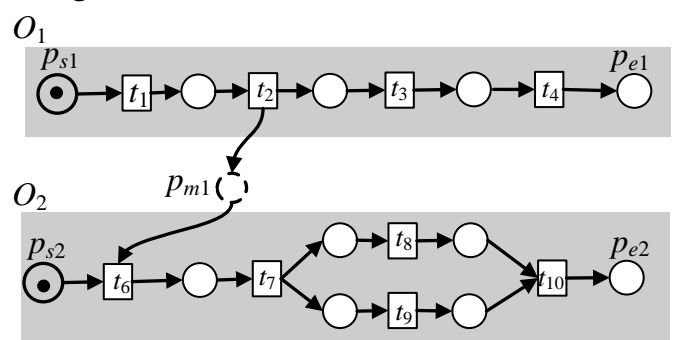

Fig. 2. An example of message exchange pattern between $O_{1}$ and $O_{2}$

\subsubsection{Resource sharing pattern}

If the required resource sets of two emergency organizations interact completely or partially, it is defined that they satisfy a resource sharing pattern. Its formal definition and RMWF-net based modeling methods are given in the next two definitions.

Definition 4.3. A resource sharing pattern is a 4-tuple $\mathrm{RSP}=\langle\mathrm{PID}$, Orgs, Mres, TID $\rangle$, where

(1) PID is the unified identifier of a pattern;

(2) Orgs represents the organizations involved in this pattern;

(3) Mres is the shared resource element; and

(4) TID is the emergency task ID that involved in this pattern.

Definition 4.4. Let $\Sigma_{\mathrm{RM} 1}=\left(P_{1}, T_{1} ; F_{1}, M_{01}\right)$ and $\Sigma_{\mathrm{RM}}=\left(P_{2}, T_{2} ; F_{2}, M_{02}\right)$ be the RMWF-net of two emergency organizations, where $P_{1}=P_{\mathrm{L} 1} \cup P_{\mathrm{R} 1} \cup P_{\mathrm{M} 1}$, $P_{2}=P_{\mathrm{L} 2} \cup P_{\mathrm{R} 2} \cup P_{\mathrm{M} 2}$. If $P_{\mathrm{R} 1} \cap P_{\mathrm{R} 2} \neq \varnothing$, then a resource sharing pattern exists between them.

Considering for example, a resource sharing pattern which is formulated as: $\left\langle\right.$ " $P_{2}$ ", $\left.\left\{O_{2}, O_{3}\right\},\left\{p_{\mathrm{r}}\right\},\left\{t_{6}, t_{11}\right\}\right\rangle$. It involves two emergency organizations $O_{2}$ and $O_{3}$, and both task $t_{6}$ and task $t_{11}$ need to access resource $p_{\mathrm{r}}$ to execute. This resource sharing pattern is shown in Fig. 3. 


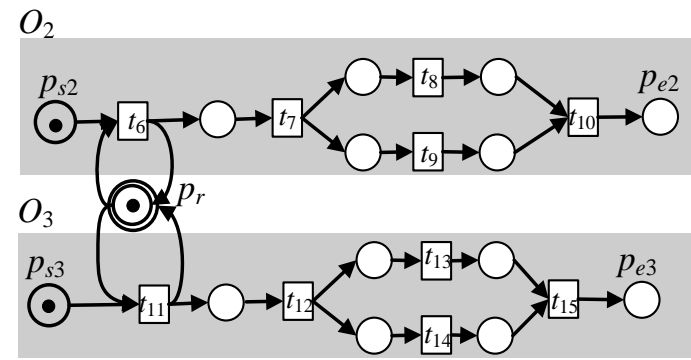

Fig. 3 An example of resource sharing pattern between $\mathrm{O}_{2}$ and $\mathrm{O}_{3}$

\subsubsection{Task synchronization pattern}

If an emergency task needs several organizations to synchronize or collaborate to accomplish it, then we define that a task synchronization pattern exists among them. Its formal definition and RMWF-net based modeling methods is given in the next two definitions.

Definition 4.5. An task synchronization pattern is a 3-tuple ASP $=\langle\mathrm{PID}$, Orgs, TID $\rangle$, where:

(1) PID is the unified identifier of a pattern;

(2) Orgs represents the organizations involved in this pattern; and

(3) TID is the emergency task ID that involved in this pattern.

Definition 4.6. Let $\Sigma_{\mathrm{RM}}=\left(P_{1}, T_{1} ; F_{1}, M_{01}\right)$ and $\Sigma_{\mathrm{RM}}=\left(P_{2}, T_{2} ; F_{2}, M_{02}\right)$ be the RMWF-net of two emergency organizations, where $P_{1}=P_{\mathrm{L} 1} \cup P_{\mathrm{R} 1} \cup P_{\mathrm{M} 1}$, $P_{2}=P_{\mathrm{L} 2} \cup P_{\mathrm{R} 2} \cup P_{\mathrm{M} 2}$. If $T_{1} \cap T_{2} \neq \varnothing$, then a task synchronization pattern exists between them.

Considering for example, a task synchronization pattern which is formulated as: “" $P_{3}$ ", $\left.\left\{O_{1}, O_{3}\right\},\left\{t_{5}\right\}\right\rangle$. It involves two emergency organizations $O_{1}$ and $O_{3}$, and task $t_{5}$ is synchronized between them. This task synchronization pattern is illustrated in Fig. 4 where the small rectangles with grids are used to represent the synchronized tasks.

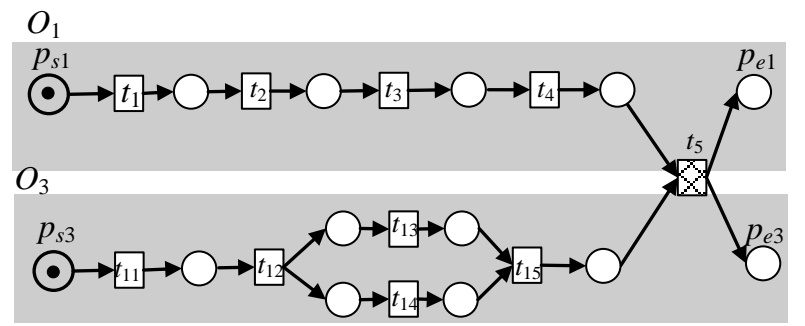

Fig. 4. An example of task synchronization pattern between $O_{1}$ and $O_{3}$

\subsection{RMWF-net based modeling of collaborative emergency response processes}

In last sub-section, we have introduced three collaboration patterns and their corresponding modeling approaches using RMWF-net. To demonstrate the modeling process in a systematic manner, we take the collaborative fire emergency 
scenario in Section 2 as an example. The RMWF-net models of the ECC, fire brigade, and hospital are obtained and shown in Table 5. Then, the collaboration patterns in this scenario are formulated in Table 6.

\begin{tabular}{|c|c|c|}
\hline Collaboration pattern & $\begin{array}{l}\text { Patten } \\
\text { ID }\end{array}$ & Formal specifications \\
\hline \multirow{4}{*}{ Message exchange pattern } & $P_{1}$ & $\left\langle “ P_{1} ",\{\right.$ ECC, Fire Brigade $\left.\},\left\{p_{\mathrm{m} 1}\right\},\left\{t_{2}\right\},\left\{t_{6}\right\}\right\rangle$ \\
\hline & $P_{2}$ & $\left\langle “ P_{2} ",\{\right.$ ECC, Hospital $\left.\},\left\{p_{\mathrm{m} 2}\right\},\left\{t_{2}\right\},\left\{t_{11}\right\}\right\rangle$ \\
\hline & $P_{3}$ & $\left\langle “ P_{3} ",\{\right.$ ECC, Fire Brigade $\left.\},\left\{p_{\mathrm{m} 3}\right\},\left\{t_{10}\right\},\left\{t_{3}\right\}\right\rangle$ \\
\hline & $P_{4}$ & $\left\langle “ P_{4} ",\{\right.$ ECC, Hospital $\left.\},\left\{p_{\mathrm{m} 4}\right\},\left\{t_{16}\right\},\left\{t_{3}\right\}\right\rangle$ \\
\hline Resource sharing pattern & $P_{5}$ & $\left\langle “ P_{5} ",\{\right.$ Hospital, Fire Brigade $\left.\},\left\{p_{\mathrm{r}}\right\},\left\{t_{6}, t_{11}\right\}\right\rangle$ \\
\hline Activity synchronization pattern & $P_{6}$ & $\left\langle “ P_{6} ",\{\mathrm{ECC}\right.$, Hospital, Fire Brigade $\left.\},\left\{t_{5}\right\}\right\rangle$ \\
\hline
\end{tabular}

Finally, by integrating the RM_WF_nets of the ECC, fire brigade, and hospital in Table 5 using these collaboration patterns summarized in Table 6, the RMWF-net model of the collaborative fire emergency response processes is obtained and shown in Fig. 5.

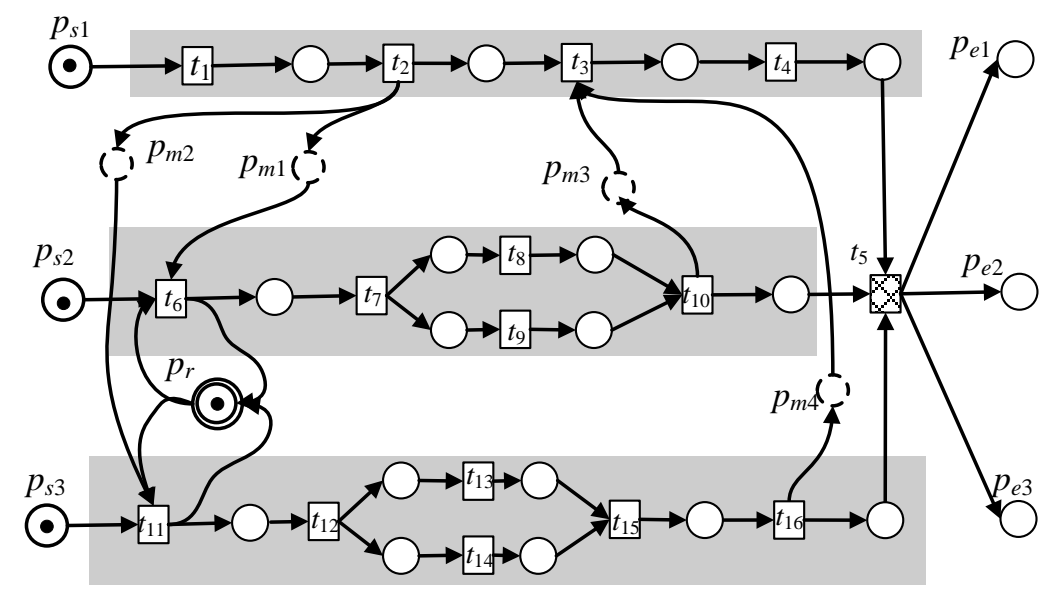

Fig. 5. RMWF-net model of the collaborative fire emergency response processes

\section{Correctness verification of the collaborative emergency response process using RMWF-net}

In this section, we formally define the correctness criteria and its corresponding verification method for the collaborative emergency response processes formulized 
in RMWF-net. Formal specification of correctness (soundness) for a WF-net is reviewed following [20].

Lemma 5.1. Let $\Sigma=\left(P, T ; F, M_{0}\right)$ be a WF-net, where $i \in P$ is the source place and $o \in P$ is the sink place, $\Sigma$ is correct if and only if :

(1) for each token in source place $i$, there will be one token generated in the sink place $o$ when finishes;

(2) if the sink place $o$ contains a token, there will no token in other places; and

(3) there are no dead transitions in $\Sigma$.

As for a collaborative emergency response process, its control structure model should satisfy the conditions in Lemma 5.1. Moreover, it should also be correct when considering the resource and message factors. Therefore, the correctness definition of a collaborative emergency response processes formulized in RMWF-net is formally defined as follows.

Definition 5.1. Let $\Sigma_{\mathrm{RM} k}=\left\{\Sigma_{\mathrm{RM} i}=\left(P_{\mathrm{RM} i}, \quad T_{\mathrm{RM} i} ; \quad F_{\mathrm{RM} i}, M_{0 \mathrm{RM} i}\right) \mid \Sigma_{\mathrm{RM} i}\right.$ is the emergency response process of the $i$ th organization $\}$ is a set of RMWF-net models representing different emergency response processes, and $\Sigma_{\mathrm{RM}}=\left(P, T ; F, M_{0}, W\right)$ is the integrated RMWF-net model of these emergency processes based on different collaboration patterns. $\Sigma_{\mathrm{RM}}$ is sound if:

(1) there is no token left in message places when a RMWF-net finishes;

(2) for each $\Sigma_{\mathrm{RM} i}$, the number of tokens in its sink place equals with the original number of the source place when it finishes. Meanwhile, there is no token in other logic places; and

(3) there are no dead transitions in $\Sigma_{\mathrm{RM}}$.

To demonstrate the verification process in detail, we consider the collaborative fire emergency response processes as an example. It is worth noting that we apply the Platform Independent Petri net Editor 3.0, PIPE 3.0 for short, to support the whole verification process. The integrated RMWF-net model in PIPE 3.0 is enacted as shown in Fig. 6, based on which the final running state is shown in Fig. 7.

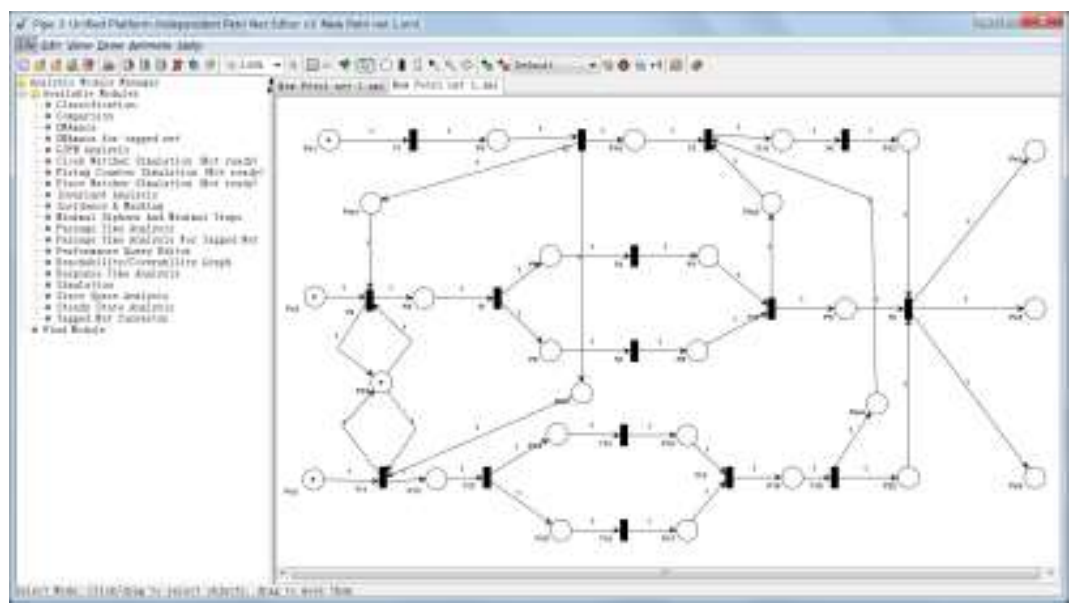

Fig. 6. Enacted RMWF-net model in PIPE 3.0 platform 
According to the final running state, we conclude that (1) there is no token left in message and logic places when this collaborative emergency response process finishes; and (2) the number of tokens in the sink place equals with the original number of the source place when the collaborative emergency response process finishes. Next, we generate the reachability graph by running the PIPE 3.0 tool as shown in Fig. 8. The graph contains 61 states, denoted as $\left\{S_{i} \mid 0 \leq i \leq 61\right\}$ where $S_{0}$ is the initial state and $S_{60}$ is the final state. It is easy to conclude that each transition appears at least one time on the path from $S_{0}$ to $S_{60}$. Thereby, there are no dead transitions in $\Sigma_{\text {RM }}$, i.e., Condition (3) in Definition 5.1 is satisfied.

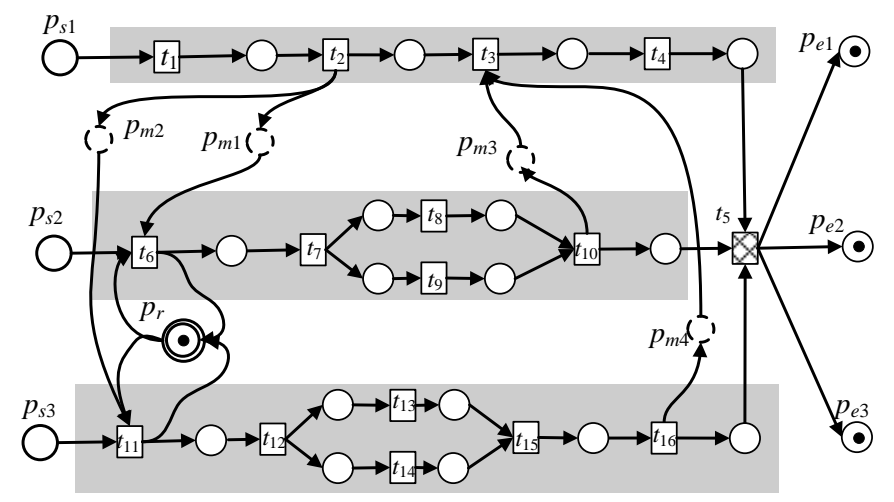

Fig. 7. Final running state of RMWF-net model in Fig. 5

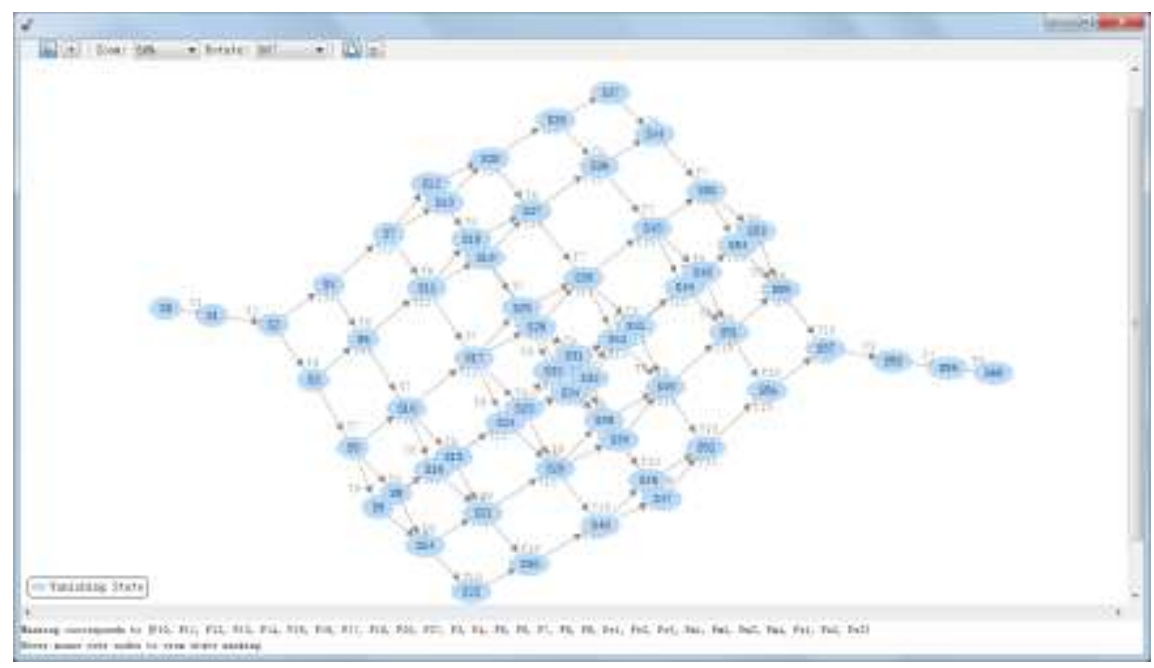

Fig. 8. Reachability graph of the RMWF-net model in Fig. 5

\section{Conclusion}

Correctness evaluation and verification of emergency response processes is becoming increasingly important to collaborative emergency response processes. Different kinds of emergency collaboration patterns exist among different 
emergency organizations, which make the modeling and verification work even more challenging. This paper gives a systematic modeling and verification approach of collaborative emergency response processes by extending Petri net with resource and message factors. Detailed modeling for collaboration emergency response processes are investigated by defining message exchange pattern, resource sharing pattern, and task synchronization pattern. Correctness of the RMWF-Net is formally defined and verified.

The paper could be deemed as a training exercise tool seeking for vulnerable and key links in the process and improve the emergency response efficiency. Collaborative fire emergency disposal is one of the most challenging problems for emergency managers and political officials. If an emergency response plan could be simulated and trained using the proposed approach after it was first established, potential conflicts in time, sequence, and resources can be identified in time. Then, they could improve the emergency response plan to reduce or eliminate these vulnerabilities. In the same way, all the participants in the emergency plan could also have a clear understanding of their emergency tasks, which will improve and enhance their job efficiency and knowledge. Generally speaking, it would be a great help to improve the reliability and validity of emergency response plan by a pretraining process. In addition, it is also could be very useful to enhance the effect of emergency drillings (or exercise). The process of emergency drillings could also be simulated and analyzed by this approach. The comparison of drilling and simulation not only could help to assess and understand the effects of good and bad drilling, but also could put forward some points that need further improvement. In short, these improvements in emergency response plan and emergency drillings have significant meaning for raising the safety level in collaborative emergency management. Currently our work only contains resource and message perspectives [27]; however, the time scale is equally important as is fully discussed in our previous work [10], [12], [22], [24]. Therefore, time performance evaluation and optimization of collaborative emergency response processes will be highly desired in the future.

Acknowledgements: This work is supported by Scientific Research Foundation of Shandong University of Science and Technology for Recruited Talents under Grant No 2014RCJJ049.

\section{References}

1. B i s r i, M. B. Examining Inter-Organizational Network during Emergency Response of West Java Earthquake 2009, Indonesia. - Procedia Environmental Sciences, Vol. 17, 2013, pp. 889-898.

2. Che n, G., X. Zh an g. Fuzzy-Based Methodology for Performance Assessment of Emergency Planning and Its Application. - Journal of Loss Prevention in the Process Industries, Vol. 22, 2009, No 2, pp. 125-132.

3. Che ng, C. Y., X. Qi a n. Evaluation of Emergency Planning for Water Pollution Incidents in Reservoir Based on Fuzzy Comprehensive Assessment. - Procedia Environmental Sciences, Vol. 2, 2010, pp. 566-570.

4. Fi edrich, F., F. Gehb a u e r, U. Rickers. Optimized Resource Allocation for Emergency Response after Earthquake Disasters. - Safety Science, Vol. 35, 2000, No 1, pp. 41-57.

5. Ford, J. K., A. M. S ch midt. Emergency Response Training: Strategies for Enhancing RealWorld Performance. - Journal of Hazardous Materials, Vol. 75, 2000, No 2, pp. 195-215. 
6. G u o, X., N. K a p u c u. Examining Coordination in Disaster Response Using Simulation Methods. - Journal of Homeland Security and Emergency Management, Vol. 12, 2015, No 4, pp. 891-914.

7. H e, Q., et al., Development and Application of a Water Pollution Emergency Response System for the Three Gorges Reservoir in the Yangtze River. - Journal of Environmental Sciences, Vol. 23, 2011, No 4, pp. 595-600.

8. K a r a gi a n n i s, G. M., E. Pi a ty s z e k, J. M. F l a u s. Industrial Emergency Planning Modeling: A First Step toward a Robustness Analysis Tool. - Journal of Hazardous Materials, Vol. 181, 2010, No 1, pp. 324-334.

9. Le e, S. J., P. H. S e o n g. Development of Automated Operating Procedure System Using Fuzzy Colored Petri Nets for Nuclear Power Plants. - Annals of Nuclear Energy, Vol. 31, 2004, No 8, pp. 849-869.

10. Li, Q., Y. D en g, C. Li u, Q. Z e n g, Y. L u. Modeling and Analysis of Subway Fire Emergency Response: An Empirical Study. - Safety Science, Vol. 84, 2016, pp. 171-180.

11. Li u, C., et al. Invariant Decomposition Conditions for Petri Nets Based on the Index of Transitions. - Information Technology Journal, Vol. 11, 2012, pp. 768-774.

12. Li u, C., Q. Z en g, H. D u a n, M. Zhou, F. Lu, J. Cheng. E-Net Modeling and Analysis of Emergency Response Processes Constrained by Resources and Uncertain Durations. - IEEE Transactions on Systems, Man, and Cybernetics: Systems, Vol. 45, 2015, No 1, pp. 84-96.

13. L u m b ro s o, D., K. S t o n e, F. V in e t. An Assessment of Flood Emergency Plans in England and Wales, France and the Netherlands. - Natural Hazards, Vol. 58, 2001, No 1, pp. 341-363.

14. M e n g, D., Q. Z e n g, F. L u, J. S u n, J. A n. Cross-Organization Task Coordination Patterns of Urban Emergency Response Systems. - Information Technology Journal, Vol. 10, 2001, No 2, pp. 367-375.

15. Murata, T. Petri Nets: Properties, Analysis and Applications. - Proceedings of the IEEE, Vol. 77, 1989, No 4, pp. 541-580.

16. Liu, C., Q. Zeng, H. Duan. Formulating the Data-Flow Modeling and Verification for Workflow: A Petri Net Based Approach. - International Journal of Science and Engineering Applications, Vol. 3, 2014, No 4, pp. 107-112.

17. Zeng Q., F. Lu, C. Li u et al. Modeling and Analysis for Cross-Organizational Emergency Response Systems Using Petri Net. - Chin. J. Comput., Vol. 36, 2013, pp. 2291-2301.

18. Re is ig, W. Petri Nets: An Introduction. Monographs in Theoretical Computer Science: An EATCS Series. Vol. 4, Berlin, Springer-Verlag, 1985.

19. T s e n g a, J., M. Li u b, R. C h an g, J. S u d, C. S h u a. Emergency Response Plan of Chlorine Gas for Process Plants in Taiwan. - Journal of Loss Prevention in the Process Industries, Vol. 21, 2008, No 4, pp. 393-399.

20. V a n D e r A a l s t, W. M. P. The Application of Petri Nets to Workflow Management. - Journal of Circuits, Systems, and Computers, Vol. 8, 1998, No 1, pp. 21-66.

21. W a n g, G. L., Y. L. Lu, J. X u. Application of GIS Technology in Chemical Emergency Response. - Journal of Environmental Science, Vol. 12, 2000, No 2, pp. 172-177.

22. Zeng, Q., C. Liu, H. Duan. Resource Conflict Detection and Removal Strategy for Nondeterministic Emergency Response Processes Using Petri Nets. - Enterprise Information Systems, 2015, pp. 1-22.

23. Z h o u, J. Petri Net Modeling for the Emergency Response to Chemical Accidents. - Journal of Loss Prevention in the Process Industries. Vol. 26, 2013, No 4, pp. 766-770.

24. Li u, C., J. C h e n g, Y. W a n g, S. G a o. Time Performance Optimization and Resource Conflicts Resolution for Multi-Project Management. - IEICE Transactions on Information and Systems, Vol. E99-D, 2016, No 3, pp. 650-660.

25. Z e n g, Q., F. L u, C. L i u, H. D u a n, C. Z h o u. Modeling and Verification for Cross-Department Collaborative Business Processes Using Extended Petri Nets. - IEEE Transactions on Systems, Man, and Cybernetics: Systems, Vol. 45, 2015, No 2, pp. 349-362.

26. Liu, C., Q. Zeng, H. Duan, F. Lu. Petri Net Based Behavior Description of CrossOrganization Workflow with Synchronous Interaction Pattern. - In: Process-Aware Systems. Vol. 495. Berlin, Heidelberg, Springer, 2014, pp. 1-10. 
27. Zeng, Q., S. X. Su n, H. Du a n, C. Li u, H. W a n g. Cross-Organizational Collaborative Workflow Mining from a Multi-Source Log. - Decision Support Systems, Vol. 54, 2013, No 3, pp. 1280-1301.

28. Cheng, J., C. Liu, M. Zhou, Q. Zeng, A. Y la-Jaaski. Automatic Composition of Semantic Web Services Based on Fuzzy Predicate Petri Nets. - IEEE Transactions on Automation Science and Engineering, Vol. 12, 2015, No 2, pp. 680-689.

29. H u a, R., Y. F u, J. Z. Y u, C. Li u. Petri Net-Based Modeling and Verification of Automatic Train Speed Control System. - Int. J. of Applied Mechanics and Materials, Vol. 571, 2014, pp. 395-399. 